\title{
Prospects of newly detecting nearby star-forming galaxies by the Cherenkov Telescope Array
}

\author{
Naoya Shimono, ${ }^{1 \star}$ Tomonori Totani, ${ }^{1,2}$ Takahiro Sudoh ${ }^{1}$ \\ ${ }^{1}$ Department of Astronomy, the University of Tokyo, 7-3-1 Hongo, Tokyo 113-0033, Japan \\ ${ }^{2}$ Research Center for the Early Universe, the University of Tokyo, 7-3-1 Hongo, Tokyo 113-0033, Japan
}

Accepted XXX. Received YYY; in original form ZZZ

\begin{abstract}
Prospects of the Cherenkov Telescope Array (CTA) for the study of very high energy gammaray emission from nearby star-forming galaxies are investigated. In the previous work, we constructed a model to calculate luminosity and energy spectrum of pion-decay gamma-ray emission produced by cosmic-ray interaction with the interstellar medium (ISM), from four physical quantities of galaxies [star formation rate (SFR), gas mass, stellar mass, and effective radius]. The model is in good agreement with the observed $\mathrm{GeV}-\mathrm{TeV}$ emission of several nearby galaxies. Applying this model to nearby galaxies that are not yet detected in $\mathrm{TeV}$ (mainly from the KINGFISH catalog), their hadronic gamma-ray luminosities and spectra are predicted. We identify galaxies of the highest chance of detection by CTA, including NGC 5236, M33, NGC 6946, and IC 342. Concerning gamma-ray spectra, NGC 1482 is particularly interesting because our model predicts that this galaxy is close to the calorimetric limit and its gamma-ray spectral index in $\mathrm{GeV}-\mathrm{TeV}$ is close to that of cosmic-ray protons injected into ISM. Therefore this galaxy may be detectable by CTA even though its GeV flux is below the Fermi Large Area Telescope sensitivity limit. In the $\mathrm{TeV}$ regime, most galaxies are not in the calorimetric limit, and the predicted $\mathrm{TeV}$ flux is lower than that assuming a simple relation between the TeV luminosity and SFR of M82 and NGC 253, typically by a factor of 15 . This means that a more sophisticated model beyond the calorimetric limit assumption is necessary to study $\mathrm{TeV}$ emission from star-forming galaxies.
\end{abstract}

Key words: gamma-rays: galaxies - galaxies: starburst

\section{INTRODUCTION}

All galaxies with substantial star formation activity (star-forming galaxies, SFGs) are gamma-ray emitters. Gamma rays are produced by the decay of pions, which are produced by collisions of cosmicray protons accelerated by supernova remnants with the interstellar medium (ISM). This process is dominant in the diffuse Galactic gamma-ray background radiation (Abdo et al. 2009). Observing this gamma-ray emission from extragalactic galaxies is not easy because of smaller luminosities compared with those of active galactic nuclei (AGNs) such as blazars, but in recent years an increasing number of nearby SFGs [the Large and Small Magellanic Clouds (LMC and SMC), M31, NGC 253, M82, NGC 4945, NGC 1068, NGC 2146, Arp 220, Arp 299, and M33] have been detected in the $\mathrm{GeV}$ band by the Fermi Large Area Telescope (LAT) (Abdo et al. 2010a,b,c,d; Ackermann et al. 2012b; Tang et al. 2014; Peng et al. 2016; Lopez et al. 2018; Ajello et al. 2020; Xi et al. 2020a,b), proving that gamma-ray emission from SFGs is indeed ubiquitous. M82 and NGC 253 were detected also in the TeV band by ground-based

^ E-mail: shimono@astron.s.u-tokyo.ac.jp air Cherenkov telescopes (Acero et al. 2009; VERITAS Collaboration et al. 2009; H. E. S. S. Collaboration et al. 2018), and more detections in $\mathrm{TeV}$ are expected by the Cherenkov Telescope Array (CTA) project (Cherenkov Telescope Array Consortium et al. 2019). A large sample of gamma-ray emitting SFGs in GeV-TeV in the near future will be useful to study the production and propagation of cosmic rays in various types of galaxies and to study high-energy background radiation. It is then important to prepare theoretical predictions for gamma-ray luminosities and spectra of nearby SFGs.

The most popular approach to predict gamma-ray luminosity from a galaxy is to relate it with some of physical quantities of the galaxy, especially the star formation rate (SFR). Gas mass of a galaxy is often additionally used to take into account the interaction of cosmic rays with ISM (Torres 2004; Domingo-Santamaría \& Torres 2005; Persic et al. 2008; Lacki et al. 2010; Inoue 2011; Lacki et al. 2011; Wang \& Fields 2018; Ambrosone et al. 2021). In other modelings, microphysical parameters about cosmic-ray propagation (e.g. magnetic field strengths, convection velocities, or mean free paths) are introduced as free parameters and these are fit to reproduce the observed gamma-ray luminosity of a galaxy (Yoast-Hull 
et al. 2013, 2014; Eichmann \& Becker Tjus 2016; Peretti et al. 2019; Krumholz et al. 2020). In a more detailed approach, cosmic-ray production and propagation are numerically simulated within a galaxy, though it is difficult to adopt such an approach to predict gamma-ray emission from a variety of nearby galaxies (Martin 2014; Pfrommer et al. 2017; Chan et al. 2019).

In a previous work, we constructed a model to predict gammaray luminosity and spectrum of a SFG, from four input parameters (SFR, gas mass, stellar mass, and effective radius) that are easily available for nearby galaxies [Sudoh et al. (2018), hereafter S18]. The escape time scale of cosmic-ray protons is estimated as a function of the proton energy, considering diffusion in turbulent magnetic fields and advection. It was found that this model reproduces the observed gamma-ray luminosities of nearby galaxies better than those using only SFR and/or gas mass of galaxies. In this paper, we apply the S18 model to nearby galaxies that are not yet detected in the $\mathrm{TeV}$ band (mainly from the KINGFISH catalog), predict their gamma-ray emission properties, and discuss their relation to galactic physical parameters. Then the prospect of future studies with CTA will be discussed.

In Section 2 our model is described, especially about some minor changes from the original S18 model. The nearby galaxy sample studied here will be presented in Section 3. Then results are presented in Section 4, followed by conclusions in Section 5.

\section{MODEL}

We use the model constructed by S18. In this model, the proton injection spectrum is formulated as:

$$
\frac{d N}{d t d E_{p}}=C\left(\frac{\psi}{\mathrm{M}_{\odot} \mathrm{yr}^{-1}}\right)\left(\frac{E_{p}}{\mathrm{GeV}}\right)^{-\Gamma_{\mathrm{inj}}}
$$

where $\psi$ is star formation rate, $\Gamma_{\text {inj }}$ is proton injection index, and $C$ is the parameter determined by fitting the model to the observed $\mathrm{GeV}$ luminosities. We set the maximum energy of proton energy as $4 \times 10^{15} \mathrm{eV}$, which corresponds to the knee. This choice does not affect our results, because its effect starts to appear at the energy range where intergalactic absorption by extragalactic background light (EBL) is important (see below). Then by a one-zone modeling assuming disk geometry, we consider the probability of cosmic-ray proton collisions with ISM as a balance between collision timescale and escape timescale: $f=1-\exp \left(-t_{\mathrm{esc}} / t_{\mathrm{pp}}\right)$. Collision timescale, $t_{\mathrm{pp}}$, is expressed as $t_{\mathrm{pp}}=\left(n_{\mathrm{gas}} \sigma_{\mathrm{pp}} c\right)^{-1}$, where $n_{\mathrm{gas}}$ is ISM gas density and $\sigma_{\mathrm{pp}}$ is the pp cross-section (Kelner et al. 2006). The escape time scale of cosmic-ray protons is calculated as the minimum of advection timescale and diffusion timescale: $t_{\mathrm{esc}}=\min \left(t_{\mathrm{diff}}, t_{\mathrm{adv}}\right)$. The advection timescale is estimated from the disc scale height and velocity dispersion assuming gravitational equilibrium: $t_{\mathrm{adv}}=H_{\mathrm{g}} / \sigma$ and $G \Sigma=\sigma^{2} /\left(2 \pi H_{\mathrm{g}}\right)$, where $H_{\mathrm{g}}$ is scale height [estimated from the assumption that it is proportional to effective radius and MW values $R_{\mathrm{eff}, \mathrm{MW}}=6.0 \mathrm{kpc}$ (Sofue et al. 2009) and $H_{\mathrm{g}, \mathrm{MW}}=150 \mathrm{pc}$ (Mo et al. 2010)], $\sigma$ is the escape velocity and $\Sigma=\left(M_{\mathrm{star}}+M_{\mathrm{gas}}\right) /\left(\pi R_{\mathrm{eff}}^{2}\right)$ is surface density (Mo et al. 2010). The diffusion coefficient and timescale are estimated in a standard manner based on the Larmor radius $R_{L}$ of a proton and the coherence length $l_{0}$ of interstellar magnetic field assuming Kolmogorov-type turbulence. The diffusion coefficient is formulated as

$$
D= \begin{cases}\frac{c l_{0}}{3}\left[\left(\frac{R_{L}}{l_{0}}\right)^{\frac{1}{3}}+\left(\frac{R_{L}}{l_{0}}\right)^{2}\right] & \left(R_{L} \leq \sqrt{H_{\mathrm{g}} l_{0}}\right) \\ \frac{c H_{g}}{3} & \left(R_{L}>\sqrt{H_{\mathrm{g}} l_{0}}\right)\end{cases}
$$

(Aloisio \& Berezinsky 2004). We assume $l_{0}=\min \left(30 \mathrm{pc}, H_{\mathrm{g}}\right.$ ). Magnetic field strength is estimated by equipartition with the ISM energy density provided by star formation activity: $B^{2} /(8 \pi)=$ $\eta E_{\mathrm{SN}} r_{\mathrm{SN}} t_{\mathrm{adv}} / V$, where $E_{\mathrm{SN}}$ is the kinetic energy of a supernova, $r_{\mathrm{SN}}$ the event rate of supernova, and $V=2 \pi R_{\mathrm{eff}}^{2} H_{\mathrm{g}}$. The parameter $\eta$ is determined so that it reproduces the observed galactic magnetic field strength $B=6 \mu \mathrm{G}$ for MW (Beck 2008). From those estimations, the gamma-ray luminosity and spectrum of a model galaxy are obtained.

Absorption of gamma-rays by EBL is taken into account in our model calculations, by using the optical depth model of Inoue et al. (2013). We do not consider absorption within a galaxy in our calculation, because these do not have large effects on our results for normal SFGs up to a few TeV. Pair-production optical depth becomes about unity around $5 \mathrm{TeV}$ or higher for NGC 253 and M82 (Inoue 2011; Lacki \& Thompson 2013), and photons beyond this energy may be suppressed compared with our model predictions. Pair-production optical depth in Arp 220 (an ultra-luminous infrared galaxy) is even larger than those for NGC 253 and M82 (Lacki \& Thompson 2013), but no galaxy in our sample is as luminous in infrared as Arp 220. Absorbed gamma-rays produce secondary cascade emission by reprocessed photons at a lower energy band, but this effect is ignored in our calculation, because this component is not large in the $\mathrm{TeV}$ band compared with direct emission (Inoue 2011).

The overall normalization parameter $C$ is determined by fitting to the observed gamma-ray luminosities of nearby galaxies, which are listed in Table 1 . This is the only free parameter in this fitting, and the resultant value is $C=2.1 \times 10^{45} \mathrm{~s}^{-1} \mathrm{erg}^{-1}$ when we assume $\Gamma_{\text {inj }}=2.2$ (see below). This value is reasonable compared with a theoretical expectation, which is $C=3.2 \times 10^{45} \mathrm{~s}^{-1} \mathrm{erg}^{-1}$ when we assume the Salpeter initial mass function (IMF), a supernova mass threshold of $8 M_{\odot}, 10 \%$ of supernova explosion energy $\left(10^{51}\right.$ erg) going into cosmic-rays, and $\Gamma_{\text {inj }}=2.2$ above $10 \mathrm{GeV}$. The sample of Table 1 includes all star-forming galaxies detected by Fermi-LAT at the time of S18. Note that galaxies whose AGN might contribute to gamma-ray emission are removed (Ackermann et al. 2012b; Yoast-Hull et al. 2017; Xi et al. 2020b), and that M31 is also removed because its gamma-ray emitting region is different from gas distribution, implying that the interaction between cosmic rays and ISM is not the main source of gamma rays from this galaxy (Ackermann et al. 2017). After S18, gamma-ray detection has been reported for M33 (Ajello et al. 2020; Xi et al. 2020a,b), along with a few more galaxies whose gamma rays might originate from AGN activities. We do not include this galaxy in the fitting, but the gamma-ray luminosity predicted by our model will be compared with these observations later.

In this paper, some physical parameters of galaxies in Table 1 are modified from those in S18. For the consistency with the KINGFISH catalog (see §3), SFR, gas mass, and stellar mass except for those of MW are derived in the same way as Rémy-Ruyer et al. (2014, 2015). SFRs are derived from $\mathrm{H} \alpha$ and IR luminosities using eq. (11) in Kennicutt et al. (2009), and IR luminosities are estimated from eq. (5) in Dale \& Helou (2002) using the IRAS data (Sanders et al. 2003). Gas mass is the sum of neutral and molecular hydrogen masses. Neutral hydrogen mass is derived from $21-\mathrm{cm}$ line flux, and $\mathrm{H}_{2}$ mass is derived from $\mathrm{CO}(1-0)$ line flux assuming a metallicitydependent conversion factor $\left(X_{\mathrm{CO}-\mathrm{H}_{2}} \propto Z^{-2}\right)$ (Rémy-Ruyer et al. 2014). See e.g. Obreschkow \& Rawlings (2009) for equations of gas mass derivation. Stellar mass is estimated using the equation derived in Eskew et al. (2012) using the Spitzer data (Dale et al. 2009). 
Table 1. Physical properties of GeV-detected galaxies used to determine parameter $C$.

\begin{tabular}{lcccccc}
\hline Objects & $\begin{array}{c}D^{a} \\
(\mathrm{Mpc})\end{array}$ & $\begin{array}{c}L_{\gamma}(0.1-800 \mathrm{GeV})^{b} \\
\left(10^{39} \mathrm{erg} \mathrm{s}^{-1}\right)\end{array}$ & $\begin{array}{c}\psi^{c} \\
\left(\mathrm{M}_{\odot} \mathrm{yr}^{-1}\right)\end{array}$ & $\begin{array}{c}M_{\mathrm{gas}}{ }^{d} \\
\left(10^{9} \mathrm{M}_{\odot}\right)\end{array}$ & $\begin{array}{c}M_{\text {star }}{ }^{e} \\
\left(10^{9} \mathrm{M}_{\odot}\right)\end{array}$ & $\begin{array}{c}R_{\mathrm{eff}} f \\
\mathrm{kpc}\end{array}$ \\
\hline \hline MW & & $0.82 \pm 0.27$ & 2.6 & 4.9 & 50 & 6.0 \\
LMC & 0.05 & $0.032 \pm 0.001$ & 0.3 & 0.59 & 1.8 & 2.2 \\
SMC & 0.06 & $0.0125 \pm 0.0005$ & 0.043 & 0.46 & 0.3 & 0.7 \\
NGC 253 & 3.5 & $13 \pm 1$ & 3.3 & 3.2 & 54.4 & 0.5 \\
M82 & 3.3 & $14.7 \pm 0.7$ & 4.4 & 4.7 & 21.9 & 0.3 \\
NGC 2146 & 17.2 & $81.4 \pm 14.2$ & 11.4 & 10.4 & 87.1 & 1.7 \\
\hline
\end{tabular}

$a$ Distances taken from Kennicutt et al. (2008) for LMC, SMC and NGC 2146, Rekola et al. (2005) for NGC 253, and Foley et al. (2014) for M82.

$b$ Gamma-ray luminosities taken from Ajello et al. (2020) except for MW from Ackermann et al. (2012b). Note that the energy range is 0.1-100 GeV for MW but $0.1-800 \mathrm{GeV}$ for all other galaxies.

c Star formation rates calculated from Diehl et al. (2006); Makiya et al. (2011) for MW, and Sanders et al. (2003); Kennicutt et al. (2008, 2009) for all other galaxies. See also text.

$d$ Total gas masses (atomic plus molecular hydrogens) calculated from Paladini et al. (2007); Rémy-Ruyer et al. (2014) for MW, Staveley-Smith et al. (2003); Israel (1997); Marble et al. (2010) for LMC, Stanimirovic et al. (1999); Israel (1997); Marble et al. (2010) for SMC, Springob et al. (2005); Knudsen et al. (2007); Pilyugin et al. (2014) for NGC 253, Chynoweth et al. (2008); Weiß et al. (2005); Moustakas et al. (2010) for M82, and Rémy-Ruyer et al. (2014); Young et al. (1989) for NGC 2146.

e Stellar masses taken from Bland-Hawthorn \& Gerhard (2016) for MW, and Dale et al. (2009); Eskew et al. (2012) for other galaxies.

$f$ Effective radii taken from Sofue et al. (2009) for MW, van der Marel (2006) for LMC, Gonidakis et al. (2009) for SMC, Strickland et al. (2004) for NGC 253 and M82, and Young et al. (1988) for NGC 2146.

Figure 1 shows the comparison of our model and the observed gamma-ray luminosities by Fermi-LAT. Although NGC 2146 shows a slightly bad fit, our model is in good agreement with observations as a whole, and performs better than the simpler assumptions such as $L_{\gamma}(0.1-800 \mathrm{GeV}) \propto \psi$ (the calorimetric limit) or $L_{\gamma}(0.1-800 \mathrm{GeV})$ $\propto \psi M_{\text {gas }}$ (intended to be the escape limit, though $M_{\text {gas }}$ should be ISM density in reality). This is because we estimate the fraction of confined protons by considering more detailed physical conditions than simple models. The data point for M33, which was not used to determine $C$, is also shown here, and the luminosity predicted by our model is in reasonable agreement with the newly reported observed luminosity. The observed luminosity of NGC 2146 is explained if we assume the calorimetric limit, which indicates that the emission region of this galaxy might be much smaller than our assumption, or perhaps there is another emission component.

Because NGC 253 and M82 have been detected in the TeV band, we show our model spectra $E_{\gamma} d F_{\gamma} / d E_{\gamma}$, where $d F_{\gamma} / d E_{\gamma}$ is differential energy flux per unit photon energy, in comparison with the observed data of these galaxies in Figure 2 to verify that our model works not only for $\mathrm{GeV}$ luminosity but also for $\mathrm{GeV}-\mathrm{TeV}$ spectrum. In comparison, sensitivity curves of TeV telescopes 12 and Fermi-LAT ${ }^{3}$ are shown. The Fermi-LAT sensitivity depends on the source position on sky, and in this paper we adopt one of the four sensitivity curves at $(l, b)=\left(0^{\circ}, 0^{\circ}\right),\left(0^{\circ}, 30^{\circ}\right),\left(0^{\circ}, 90^{\circ}\right)$ and $\left(45^{\circ}, 120^{\circ}\right)$. For each galaxy, we examine the sensitivity at its location using the sensitivity map of gamma-ray flux above 100 $\mathrm{MeV}$, and choose the closest differential sensitivity curves from the four. Our model is in good agreement with the observed spectra when we choose $\Gamma_{\text {inj }}=2.1-2.2$. It should be noted that our model spectrum for MW agrees with the observed spectrum of the diffuse

\footnotetext{
1 Holler et al. (2015)

2 https://veritas.sao.arizona.edu/about-veritas/

veritas-specifications

3 http://www.slac.stanford.edu/exp/glast/groups/canda/

lat_Performance.htm
}

Galactic gamma-ray background (Ackermann et al. 2012a) when we set $\Gamma_{\text {inj }}=2.3$ (see Fig. 2 of S18). This means that our model can be applied to a wide range of SFGs with a single spectral index $\Gamma_{\text {inj }} \sim 2.2$ for the proton injection into the ISM. Note that the change of $\Gamma_{\text {inj }}$ hardly affects the trend seen in Fig. 1, because it has a quantitatively similar effect on all galaxies, provided that a single value is used for all model galaxies.

\section{SAMPLE}

Physical parameters of the galaxy sample for this work are listed in Table 2. This sample is constructed mainly from the galaxies of KINGFISH project, which aims to understand the physics of ISM in present-day galaxies (Kennicutt et al. 2011). 61 galaxies in the nearby universe (less than $30 \mathrm{Mpc}$ ) were chosen to cover a wide range of galaxy properties and ISM environments, and their SFR, gas mass, and stellar mass are compiled by Rémy-Ruyer et al. (2014, 2015), making this sample suitable for the purpose of this study. However, we removed NGC 1377 and 1404 from the sample, because SFR and/or gas mass are not available. The total number of KINGFISH galaxies used in this study is 59 (including NGC 2146, which is detected in the $\mathrm{GeV}$ band and listed in Table 1).

KINGFISH is not a complete sample about an observed flux, and some nearby galaxies that are promising for detection by CTA may be missed. Therefore we add galaxies that may be detected by CTA from the IRAS Revised Bright Galaxy Sample (RGBS) (Sanders et al. 2003) covering all the sky. $\mathrm{H} \alpha$ luminosities are available (Kennicutt et al. 2008) for RGBS galaxies that are bright enough to be detected by CTA, allowing us to estimate SFR in the same way as the KINGFISH galaxies. We then make a rough estimate of expected gamma-ray luminosity as follows. We define the value $L_{\gamma}(x)$ as

$L_{\gamma}(x)=\left.E_{\gamma} \frac{d L_{\gamma}}{d E_{\gamma}}\right|_{x}$,

where $d L_{\gamma} / d E_{\gamma}$ is differential luminosity per unit photon energy. 

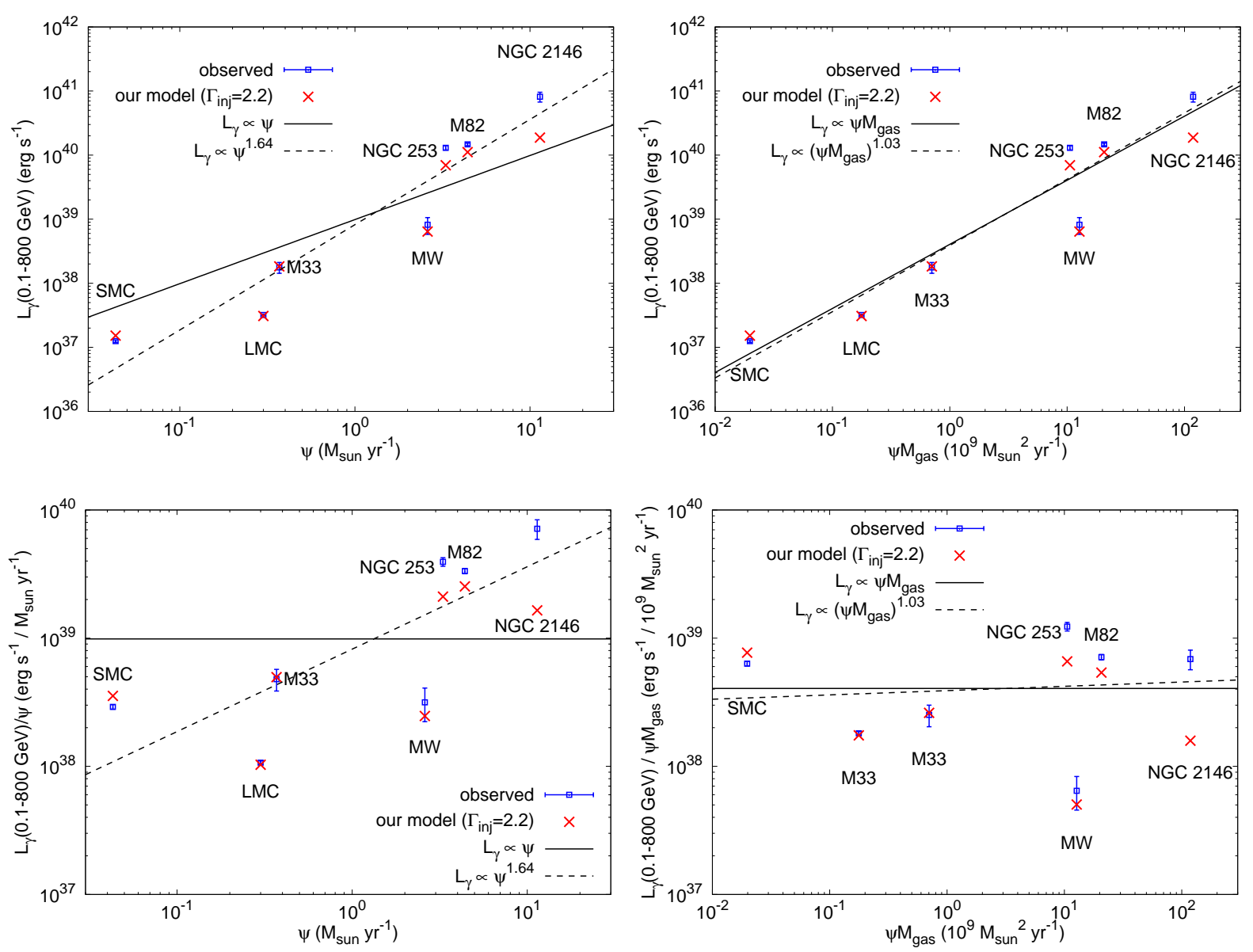

Figure 1. Upper panels: gamma-ray luminosities of nearby galaxies predicted by our model, in comparison with the observed luminosities. The left panel is shown as a function of SFR, while the right panel as a function of SFR multiplied by gas mass. The solid lines are fits by a proportional relation, $L_{\gamma}(0.1-800$ $\mathrm{GeV}) \propto \psi$ or $\psi M_{\text {gas }}$, but the dashed lines are fits with a free power-law index. It should be noted that the gamma-ray energy range is $0.1-100 \mathrm{GeV}$ for MW, while $0.1-800 \mathrm{GeV}$ for all others. Lower panels: the same as the upper panels, but the vertical axes are showing $L_{\gamma} / \psi$ and $L_{\gamma} /\left(\psi M_{\text {gas }}\right)$ to show the deviation from the simple scalings to $\psi$ or $\left(\psi M_{\mathrm{gas}}\right)$ more clearly.
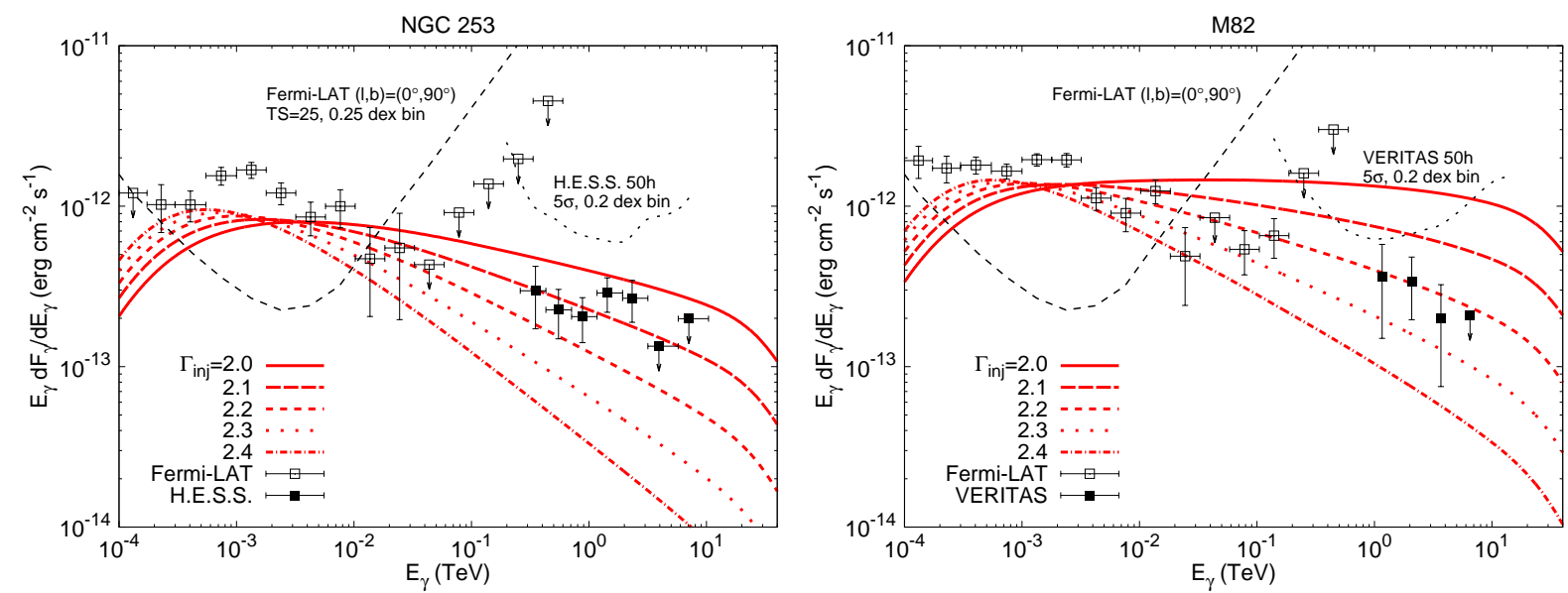

Figure 2. GeV-TeV spectra of NGC 253 (left) and M82 (right) predicted by our model, in comparison with the observed spectra by Ajello et al. 2020; H. E. S. S. Collaboration et al. 2018 for NGC 253 (10 yr for Fermi-LAT and $158 \mathrm{~h}$ for H.E.S.S) and Ajello et al. 2020; VERITAS Collaboration et al. 2009 for M82 (10 yr for Fermi-LAT and $137 \mathrm{~h}$ for VERITAS). Sensitivities of Fermi-LAT (Pass 8 Release 3 Version 2, TS $=25$ in a 0.25 dex photon energy bin) and TeV telescopes ( $5 \sigma$ detection by a $50 \mathrm{hr}$ observation in a 0.2 dex photon energy bin) are also shown. 
If we assume the same $L_{\gamma}(1 \mathrm{TeV}) / \mathrm{SFR}$ ratio as NGC 253, we can relate $\mathrm{TeV}$ gamma-ray luminosities and SFRs as

$$
\frac{L_{\gamma}(1 \mathrm{TeV})}{\mathrm{erg} / \mathrm{s}} \sim 9.1 \times 10^{37} \frac{\psi}{\mathrm{M}_{\odot} \mathrm{yr}^{-1}} .
$$

The coefficient of this equation does not change significantly (within $20 \%$ change) if we use M82 instead of NGC 253. However, there may be galaxies that have higher $L_{\gamma}(1 \mathrm{TeV}) / \mathrm{SFR}$ ratios than NGC 253. In the most optimistic scenario where cosmic-ray protons lose all the energy to $p p$ collisions, this ratio becomes $2.5 \times 10^{38}$ assuming the Salpeter IMF, the supernova mass threshold of $8 M_{\odot}$, cosmicray energy of $10^{50} \mathrm{erg}$ from a supernova, and a gamma-ray spectral index of 2.2 above $0.1 \mathrm{GeV}$.

Using this optimistic $L_{\gamma}(1 \mathrm{TeV}) / \mathrm{SFR}$ ratio to estimate $\mathrm{TeV}$ gamma-ray luminosities, we selected galaxies that are not in the KINGFISH catalog but whose expected $\mathrm{TeV}$ gamma-ray flux is brighter than the CTA sensitivity limit at $1 \mathrm{TeV}^{4}$. We then removed galaxies that are clearly AGNs or showing evidence of AGN activity (NGC 1365, NGC 1068, NGC 4945, Cen A, and Arp 299). The famous starburst galaxy Arp 220, which also shows evidence of AGN activity, is slightly below the CTA sensitivity limit by this calculation. It should be noted that NGC 1068, NGC 4945, Arp 220, and Arp 299 have been detected by Fermi-LAT and discussed in Ajello et al. (2020), but these are not included in our sample because we want to select galaxies whose gamma-ray luminosities are purely by star-forming activity. In Appendix A, we show spectra of M31 (removed due to its gas distribution; see Section 2), NGC 1068, and Arp 220 to see how our model works for the galaxies removed from our sample. We then add the eight remaining galaxies to our sample to ensure that it definitely contains all galaxies that are potentially detectable by CTA. SFR, gas mass, and stellar mass of these galaxies are derived in the same way as those of KINGFISH, and references for the necessary observed data are given in Table 2.

Among those added eight galaxies, M33 and NGC 2403 have been already detected by Fermi-LAT (Xi et al. 2020b; Ajello et al. 2020). Especially, NGC 2403 is interesting because its emission is decaying with time and this emission is coincident with SN 2004dj. However, time variability is rather marginal, and to examine whether this emission is really coming from a supernova, the estimate of steady gamma-ray flux from its star-forming activity is important.

We need effective radii of galaxies to calculate gamma-ray luminosities and spectra. We use the J-band half-light radii of the 2MASS Large Galaxy Atlas (Jarrett et al. 2003) for most galaxies in our sample. Effective radii of dwarf galaxies DDO 53, Ho I, and M81dwB are not available in the 2MASS data, and we use the results of Oh et al. (2011) for these galaxies.

\section{RESULT}

\subsection{TeV gamma-ray detectability by CTA}

In Figure 3 (left), we show the predicted fluxes at $1 \mathrm{TeV}$ by our model assuming $\Gamma_{\text {inj }}=2.2$. In the plot, these fluxes are compared with the simple expectation using Equation 4. The fluxes predicted by our model are typically 15 times lower than the simple estimates, indicating that cosmic rays responsible for $\mathrm{TeV}$ emission are less confined than those in NGC 253.

4 https://www.cta-observatory.org/science/

cta-performance/
For comparison, the same plot is made for within the FermiLAT energy band at $3 \mathrm{GeV}$ as the right panel of the same figure, in comparison with the simple estimates being proportional to SFR:

$\frac{L_{\gamma}(3 \mathrm{GeV})}{\mathrm{erg} / \mathrm{s}} \sim 4.7 \times 10^{38} \frac{\psi}{\mathrm{M}_{\odot} \mathrm{yr}^{-1}}$,

where the coefficient is again determined based on NGC 253. The fluxes predicted by our model are again lower than the simple estimates by a factor of 7 , but the differences are smaller than the $\mathrm{TeV}$ band (by a factor of 15/7), which is reasonable because of stronger confinement expected for lower energy cosmic rays. Note that M33 has been detected by Fermi-LAT as mentioned above, and Ackermann et al. (2012b) reported a signature of detection (TS 15) for NGC 5236. Other galaxies have not yet been detected by Fermi-LAT, which is consistent with our model prediction.

Table 3 presents gamma-ray fluxes $\left(E_{\gamma} d F_{\gamma} / d E_{\gamma}\right.$ at $\left.1 \mathrm{TeV}\right)$ predicted by our model for the galaxies considered in this paper, in descending order when $\Gamma_{\mathrm{inj}}=2.2$. The spectra of the top six galaxies (NGC 5236, M33, NGC 6946, IC 342, NGC 2146, and NGC 7331) are shown in Figure 4. The fluxes of these galaxies assuming $\Gamma_{\text {inj }}=2.2$ are lower than the CTA sensitivity limit (50h, $5 \sigma$ ) by a factor of a few. It should be noted that the sensitivity limit quoted here is in a photon energy bin of a 0.2 dex width, and a fainter flux can be detected if a wider energy bin width is adopted. In fact, NGC 253 has been detected by H.E.S.S, whose flux is also fainter than its sensitivity limit by a similar factor. Therefore we conclude that there is a reasonable chance of detection of these galaxies by CTA if sufficient observing time is devoted. We note that the chance of TeV gamma-ray detection for NGC 2146 is enhanced if the TeV flux is higher than that of our model by the same factor as the discrepancy at $\mathrm{GeV}$ energy range.

\subsection{Properties of TeV gamma-ray spectrum}

The power-law index $\Gamma_{\text {inj }}$ of injected proton spectrum is a free model parameter in our model, but the gamma-ray energy spectrum is calculated by the model taking into account cosmic-ray propagation and escape. Then the difference between gamma-ray and proton spectra is a good indicator of the efficiency of cosmic-ray collisions with ISM before its escape. Therefore we show the histogram of $\alpha-\Gamma_{\text {inj }}$ in Figure 5, where $\alpha$ is the photon spectral index defined as $d F_{\gamma} / d E_{\gamma} \propto E_{\gamma}^{-\alpha+1}$. We calculated $\alpha$ by a power-law fit to the modeled spectra in the range of 0.1-10 TeV. In most galaxies $\alpha-\Gamma_{\text {inj }}$ is larger than 0.2, but M82, NGC 1482 and DDO 165 have notably smaller values than other galaxies, implying that cosmic-ray interaction is particularly efficient in these.

To examine this, we made a plot of $L_{\gamma}(1 \mathrm{TeV}) / \psi$ versus $\Sigma_{\text {gas }}=M_{\text {gas }} /\left(\pi R_{\text {eff }}^{2}\right)$ (surface gas density) in Figure 6 (left panel). If $\Sigma_{\text {gas }}$ is sufficiently high, cosmic-ray collision efficiency would be high and gamma-ray emission would be close to the calorimetric limit (dashed line), and hence $L_{\gamma}(1 \mathrm{TeV}) / \psi$ will become constant asymptotically. This trend is indeed seen in the figure, and M82, NGC 1482 and DDO 165 are located at the highest $\Sigma_{\text {gas }}$ region. The spectrum of NGC 1482 predicted by our model is shown in Figure 4 (bottom left). Comparing to the CTA sensitivity, detection may not be easy but not impossible depending on model parameters and uncertainties. If detected, we predict that this galaxy would have a particularly hard spectrum compared with other SFGs. On the other hand, the predicted flux of DDO 165 is far below the CTA sensitivity limit (see Figure 3).

Gamma-ray luminosities of SFGs are often compared with 
Table 2. Physical parameters of galaxies in the sample of this work. The full table is available in supplementary material (online).

\begin{tabular}{ccccccccccc}
\hline name & $D(\mathrm{Mpc})$ & ref & $\psi\left(\mathrm{M}_{\odot} \mathrm{yr}^{-1}\right)$ & ref & $M_{\text {gas }}\left(10^{9} \mathrm{M}_{\odot}\right)$ & ref & $M_{\text {star }}\left(10^{9} \mathrm{M}_{\odot}\right)$ & ref & $R_{\text {eff }}(\mathrm{kpc})$ & ref \\
\hline \hline NGC 5236 & 4.5 & $(1)$ & 3 & $(2)(3)$ & 17 & $(4)$ & 49 & $(5)$ & 3 & $(6)$ \\
M33 & 0.84 & $(2)$ & 0.37 & $(2)(3)$ & 1.9 & $(7)(8)(9)$ & 3.2 & $(5)$ & 1.4 & $(6)$ \\
NGC 6946 & 6.8 & $(10)$ & 4.9 & $(11)$ & 21 & $(4)$ & 59 & $(11)$ & 4.5 & $(6)$ \\
IC 342 & 3.3 & $(10)$ & 1.5 & $(11)$ & 14 & $(4)$ & 81 & $(11)$ & 3.9 & $(6)$ \\
NGC 7331 & 14 & $(10)$ & 4.5 & $(11)$ & 42 & $(4)$ & 140 & $(11)$ & 3.2 & $(6)$ \\
$\ldots$ & $\cdots$ & $\cdots$ & $\ldots$ & $\cdots$ & $\cdots$ & $\cdots$ & $\cdots$ & $\cdots$ & $\cdots$ & $\cdots$ \\
\hline
\end{tabular}

References: (1) Galametz et al. (2011), (2) Kennicutt et al. (2008), (3) Sanders et al. (2003), (4) Rémy-Ruyer et al. (2014), (5) Dale et al. (2009), (6) Jarrett et al. (2003), (7) Gratier et al. (2010), (8) Heyer et al. (2004), (9) Pilyugin et al. (2014), (10) Kennicutt et al. (2011), (11) Rémy-Ruyer et al. (2015), (12) Paturel et al. (2003), (13) Allison et al. (2014), (14) Israel (1997), (15) Leroy et al. (2008), (16) Moustakas et al. (2010), (17) Madden et al. (2013), (18) Oh et al. (2011)
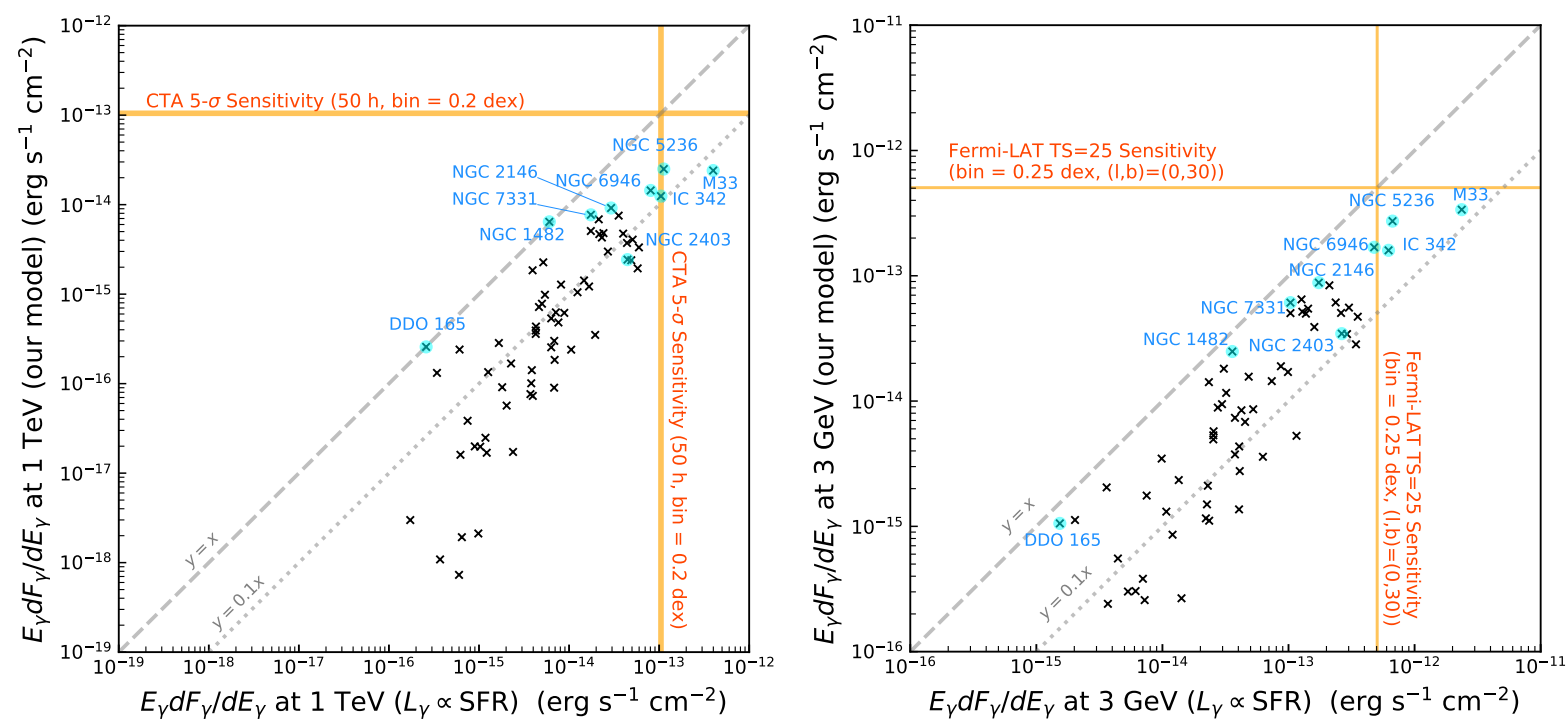

Figure 3. The fluxes at $1 \mathrm{TeV}$ (left) and $3 \mathrm{GeV}$ (right) predicted by our model assuming $\Gamma_{\text {inj }}=2.2$ are shown in the vertical axes, in comparison with the simpler prediction assuming $L_{\gamma} \propto$ SFR and the $L_{\gamma} / \mathrm{SFR}$ ratio of NGC 253 shown in the horizontal axes. Here we adopt the Fermi-LAT sensitivity at $(l, b)=\left(0^{\circ}, 30^{\circ}\right)$ that is intermediate among those of four sky locations provided at the Fermi-LAT website, and CTA South sensitivity. Blue-highlighted labeled galaxies are discussed in the text or those with spectra shown in figures.

Table 3. List of gamma-ray fluxes $\left(E_{\gamma} d F_{\gamma} / d E_{\gamma}\right.$ at $\left.1 \mathrm{TeV}\right)$ predicted by our model, in units of erg s $\mathrm{cm}^{-1}$ in the descending order when $\Gamma_{\mathrm{inj}}=2.2$. The full table is available in supplementary material (online).

\begin{tabular}{lccccc}
\hline name & $\Gamma_{\text {inj }}=2.0$ & $\Gamma_{\text {inj }}=2.1$ & $\Gamma_{\text {inj }}=2.2$ & $\Gamma_{\text {inj }}=2.3$ & $\Gamma_{\text {inj }}=2.4$ \\
\hline \hline NGC 5236 & $7.9 \times 10^{-14}$ & $4.6 \times 10^{-14}$ & $2.5 \times 10^{-14}$ & $1.3 \times 10^{-14}$ & $6.8 \times 10^{-15}$ \\
M33 & $7.6 \times 10^{-14}$ & $4.4 \times 10^{-14}$ & $2.4 \times 10^{-14}$ & $1.3 \times 10^{-14}$ & $6.6 \times 10^{-15}$ \\
NGC 6946 & $4.6 \times 10^{-14}$ & $2.6 \times 10^{-14}$ & $1.4 \times 10^{-14}$ & $7.6 \times 10^{-15}$ & $3.9 \times 10^{-15}$ \\
IC 342 & $3.9 \times 10^{-14}$ & $2.3 \times 10^{-14}$ & $1.2 \times 10^{-14}$ & $6.6 \times 10^{-15}$ & $3.4 \times 10^{-15}$ \\
NGC 2146 & $2.9 \times 10^{-14}$ & $1.7 \times 10^{-14}$ & $9.2 \times 10^{-15}$ & $4.8 \times 10^{-15}$ & $2.5 \times 10^{-15}$ \\
NGC 7331 & $2.5 \times 10^{-14}$ & $1.4 \times 10^{-14}$ & $7.7 \times 10^{-15}$ & $4.1 \times 10^{-15}$ & $2.1 \times 10^{-15}$ \\
$\ldots$ & $\ldots$ & $\ldots$ & $\ldots$ & $\ldots$ & $\ldots$ \\
\hline & & & & & \\
\hline
\end{tabular}

$\psi M_{\mathrm{gas}}$, with an expectation of $L_{\gamma} \propto \psi M_{\mathrm{gas}}$. Here, the amount of gas mass is considered as an indicator of cosmic-ray collision efficiency. To examine this, a correlation plot between $L(1 \mathrm{TeV}) / \psi$ and $M_{\text {gas }}$ is also shown in the right panel of Figure 6 . The data points show a positive correlation similar to the case of the $L(1 \mathrm{TeV}) / \psi-\Sigma_{\text {gas }}$ correlation, but as expected, the scatter is much larger because the information of galaxy sizes is not used. This indicates a limitation of using $\psi M_{\text {gas }}$ as an indicator of gamma-ray luminosity. The same correlations between $L(3 \mathrm{GeV}) / \psi$ versus $\Sigma_{\text {gas }}$ or $M_{\text {gas }}$ are shown in Figure 7. In both the energy bands, $\Sigma_{\text {gas }}$ is a good indicator of $L_{\gamma} / \psi$. Compared with the relation in $\mathrm{TeV}$, the relation in $\mathrm{GeV}$ becomes flat at $\Sigma_{\text {gas }} \gtrsim 1 \mathrm{~g} \mathrm{~cm}^{-2}$. This is expected because $\mathrm{GeV}$ emission would reach the calorimetric limit at lower $\Sigma_{\text {gas }}$. 

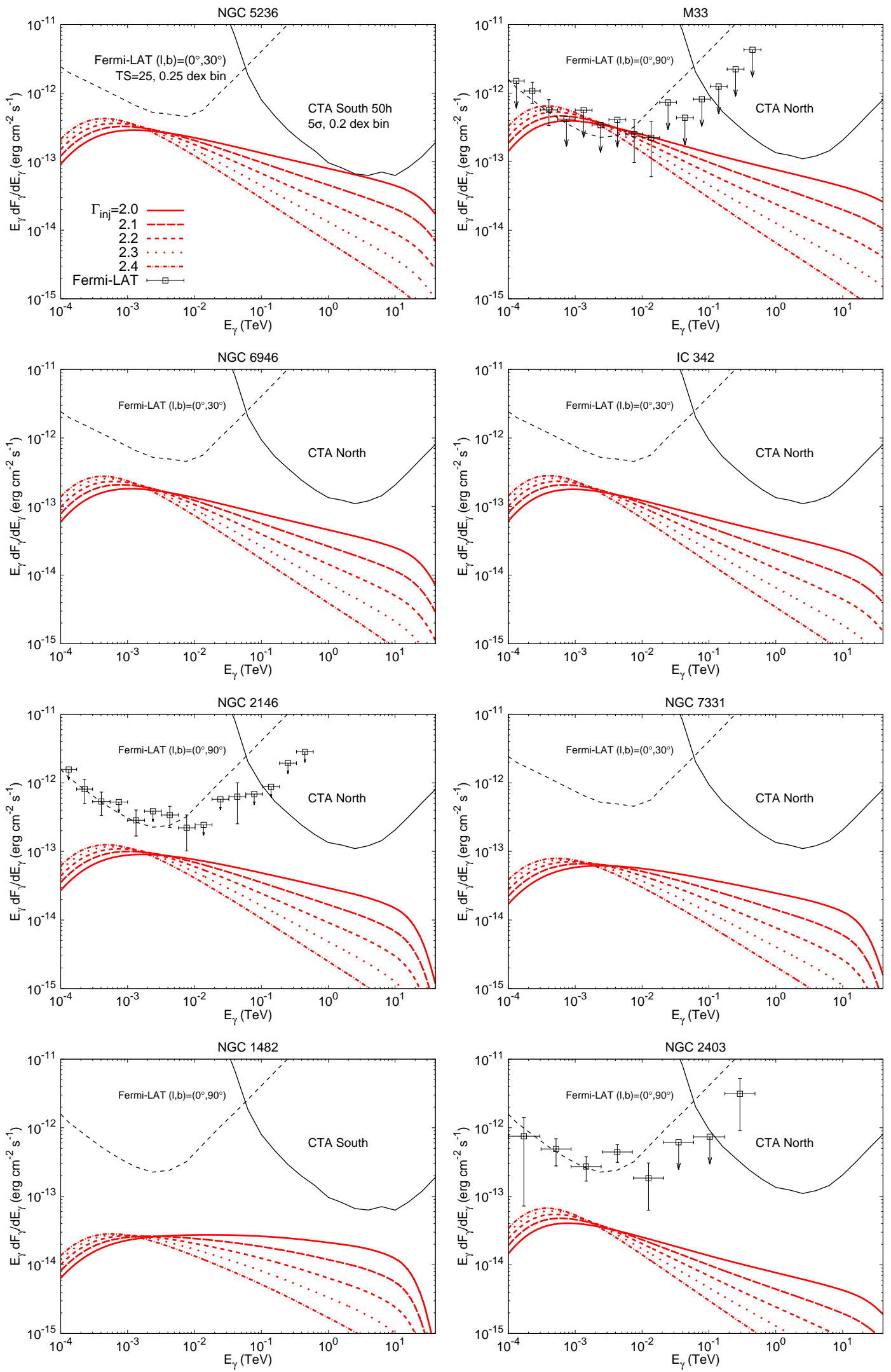

Figure 4. Examples of gamma-ray spectra of nearby galaxies predicted by our model. The sensitivities of CTA South or North are shown, depending on their declinations. NGC 5236, M33, NGC 6946, IC 342, NGC 2146, and NGC 7331 are the brightest among the sample considered in this work (except those already detected in TeV). NGC 1482 is predicted to have a particularly hard spectrum which may be detected by CTA. NGC 2403 has been detected by Fermi-LAT, but the $\mathrm{GeV}$ flux might originate from a supernova. Data points are taken from Xi et al. (2020b) for NGC 2403 (5.7 yr), and Ajello et al. (2020) (10 yr) for other galaxies. 


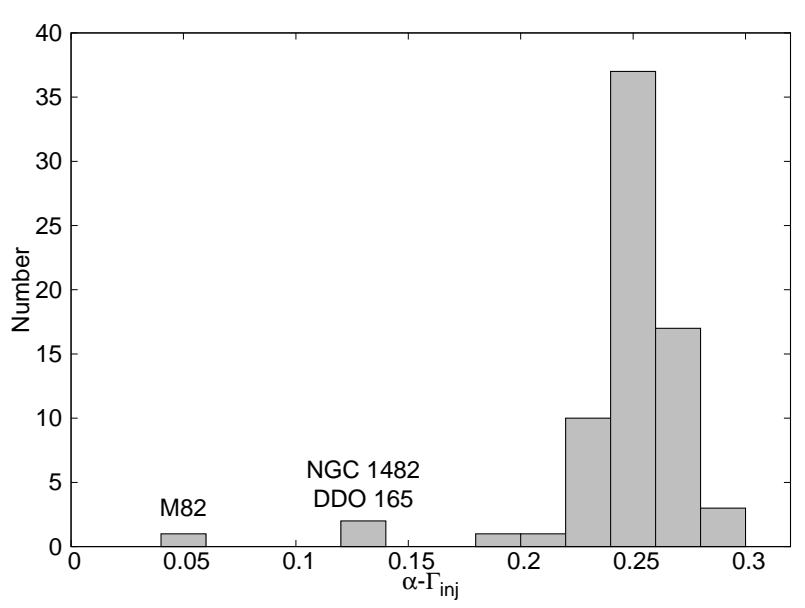

Figure 5. Histogram of $\alpha-\Gamma_{\text {inj }}$ assuming $\Gamma_{\text {inj }}=2.2$, where $\alpha$ is the powerlaw index of a photon spectrum, obtained by a fit to the modeled gamma-ray spectra of galaxies in $0.1-10 \mathrm{TeV}$.

\section{DISCUSSION \& CONCLUSIONS}

In this work, we calculated expected fluxes and spectra of $\mathrm{TeV}$ gamma rays from nearby SFGs, mainly included in the catalog of KINGFISH, based on the four physical quantities of galaxies: SFR, gas mass, stellar mass, and effective radius. Calculations were done using the S18 model, which considers energy-dependent propagation and escape of cosmic rays. These model predictions are more reliable than simpler estimates based only on SFRs. Predicted gamma-ray fluxes at $1 \mathrm{TeV}$ are tabulated in Table 3, for several values of the assumed spectral index $\Gamma_{\text {inj }}$ of cosmic-ray protons injected into ISM. Our recommendation is $\Gamma_{\text {inj }}=2.2$ because this value is close to those (2.1-2.3) favored for MW, NGC 253, and M82, though other galaxies may have a significantly different value.

Then we examined the detectability of these galaxies by CTA. There is a good chance of detection for NGC 5236, M33, NGC 6946, and IC 342. Our model predicts that power-law spectral indices of gamma-ray emission in the CTA band are typically $0.20-0.30$ softer than those of injected protons. However, some galaxies having particularly high surface gas densities, such as M82, NGC 1482 and DDO 165 , have harder spectra with a photon index softer only by at most 0.14 than protons. It is interesting that NGC 1482 may also be detected by CTA, and we predict that this galaxy would have a particularly hard $\mathrm{TeV}$ spectrum than other SFGs if the proton injection spectrum is universal. $\mathrm{TeV}$ gamma-ray luminosities of most galaxies in the sample predicted by our model are lower than the simple estimates based only on SFR and assuming the same $L_{\gamma}(1 \mathrm{TeV}) / \psi$ ratio as NGC 253 . The $L_{\gamma}(1 \mathrm{TeV}) / \psi$ ratio increases with surface gas densities of galaxies. These results indicate that most nearby galaxies are not in the calorimetric limit and cosmicray escape from galaxies is important.

Recently detection of GeV gamma rays from NGC 2403 by Fermi-LAT was reported (Xi et al. 2020a), and the gamma-ray flux seems variable. Then it was proposed that this gamma-ray emission is coming from a coincident supernova 2004dj. However, flux variability is statistically marginal, and a possibility of gammaray emission from other origins remains. Therefore we show here the gamma-ray spectrum of NGC 2403 predicted by our model in Figure 4 . The observed flux is about an order of magnitude higher than the expected flux, and it is unlikely that star-formation activity and cosmic-ray interaction explain the observed flux. Therefore the observed $\mathrm{GeV}$ flux should be explained by the supernova or any other gamma-ray emission processes.

Throughout, we have focused on gamma-ray emission produced by hadronic processes. This assumption is believed to be secure in the $\mathrm{GeV}$ range. However, recent studies have found that leptonic emission powered by pulsars may significantly contribute to, or even dominate, the diffuse Galactic gamma-ray emission in the TeV regime (Linden \& Buckman 2018; Sudoh et al. 2019). If future CTA observations detect SFGs with fluxes larger than predicted by our hadronic model, it may suggest a contribution from leptons, offering support for the importance of pulsar emission in the TeV sky.

\section{ACKNOWLEDGEMENTS}

This work was conducted in the context of the CTA Consortium. This research has made use of the CTA instrument response functions provided by the CTA Consortium and Observatory, see http://www.cta-observatory.org/science/ cta-performance/ (version prod3b-v1) for more details. TT was supported by the JSPS/MEXT KAKENHI Grant Numbers $18 \mathrm{~K} 03692$ and 17H06362. T.S. is supported by a Research Fellowship of Japan Society for the Promotion of Science (JSPS) and by JSPS KAKENHI Grant No. JP 18J20943.

\section{DATA AVAILABILITY}

The data underlying this article will be shared on reasonable request to the corresponding author. The full lists for Table 2 and Table 3 are available in their online supplementary materials.n

\section{REFERENCES}

Abdo A. A., et al., 2009, Phys. Rev. Lett., 103, 251101

Abdo A. A., et al., 2010a, A\&A, 512, A7

Abdo A. A., et al., 2010b, A\&A, 523, L2

Abdo A. A., et al., 2010c, A\&A, 523, A46

Abdo A. A., et al., 2010d, ApJ, 709, L152

Acero F., et al., 2009, Science, 326, 1080

Ackermann M., et al., 2012a, ApJ, 750, 3

Ackermann M., et al., 2012b, ApJ, 755, 164

Ackermann M., et al., 2017, ApJ, 836, 208

Adams T. F., Weedman D. W., 1975, ApJ, 199, 19

Ajello M., Di Mauro M., Paliya V. S., Garrappa S., 2020, ApJ, 894, 88

Allison J. R., Sadler E. M., Meekin A. M., 2014, MNRAS, 440, 696

Aloisio R., Berezinsky V., 2004, ApJ, 612, 900

Ambrosone A., Chianese M., Fiorillo D. F. G., Marinelli A., Miele G., Pisanti O., 2021, MNRAS,

Armus L., Heckman T. M., Miley G. K., 1990, ApJ, 364, 471

Beck R., 2008, in Aharonian F. A., Hofmann W., Rieger F., eds, American Institute of Physics Conference Series Vol. 1085, American Institute of Physics Conference Series. pp 83-96 (arXiv:0810.2923), doi:10.1063/1.3076806

Bland-Hawthorn J., Gerhard O., 2016, ARA\&A, 54, 529

Braun R., Thilker D. A., Walterbos R. A. M., Corbelli E., 2009, ApJ, 695, 937

Brown M. J. I., et al., 2014, ApJS, 212, 18

Chan T. K., Kereš D., Hopkins P. F., Quataert E., Su K. Y., Hayward C. C., Faucher-Giguère C. A., 2019, MNRAS, 488, 3716 

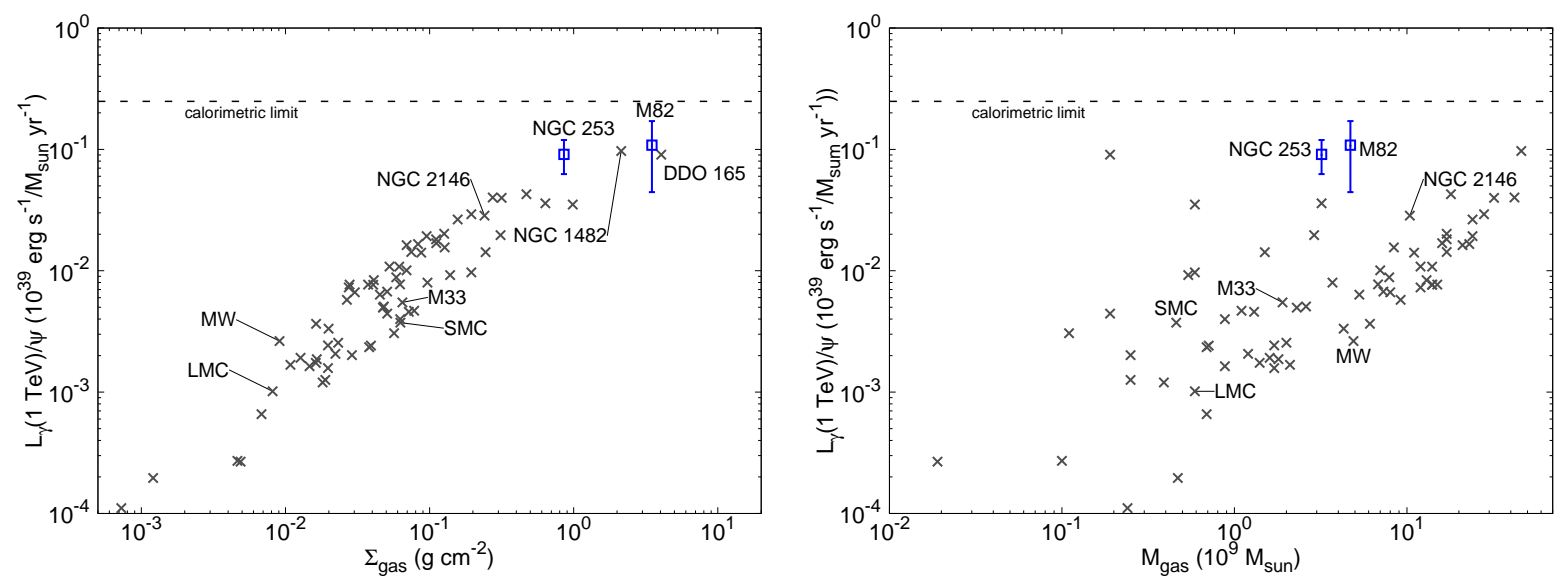

Figure 6. Correlation between $L_{\gamma}(1 \mathrm{TeV}) / \psi$ and gas surface density (left), or gas mass (right), of nearby galaxies predicted by our model assuming $\Gamma_{\text {inj }}=2.2$. The dashed line is the calorimetric limit, $L_{\gamma}\left(1 \mathrm{TeV}\right.$ ) $/ \mathrm{SFR} \sim 2.5 \times 10^{38} \mathrm{erg} \mathrm{s}^{-1} / \mathrm{M}_{\odot} \mathrm{yr}^{-1}$ (see Section 3). Blue points are galaxies detected by TeV gamma-ray telescopes based on the observations by H. E. S. S. Collaboration et al. (2018) and VERITAS Collaboration et al. (2009).
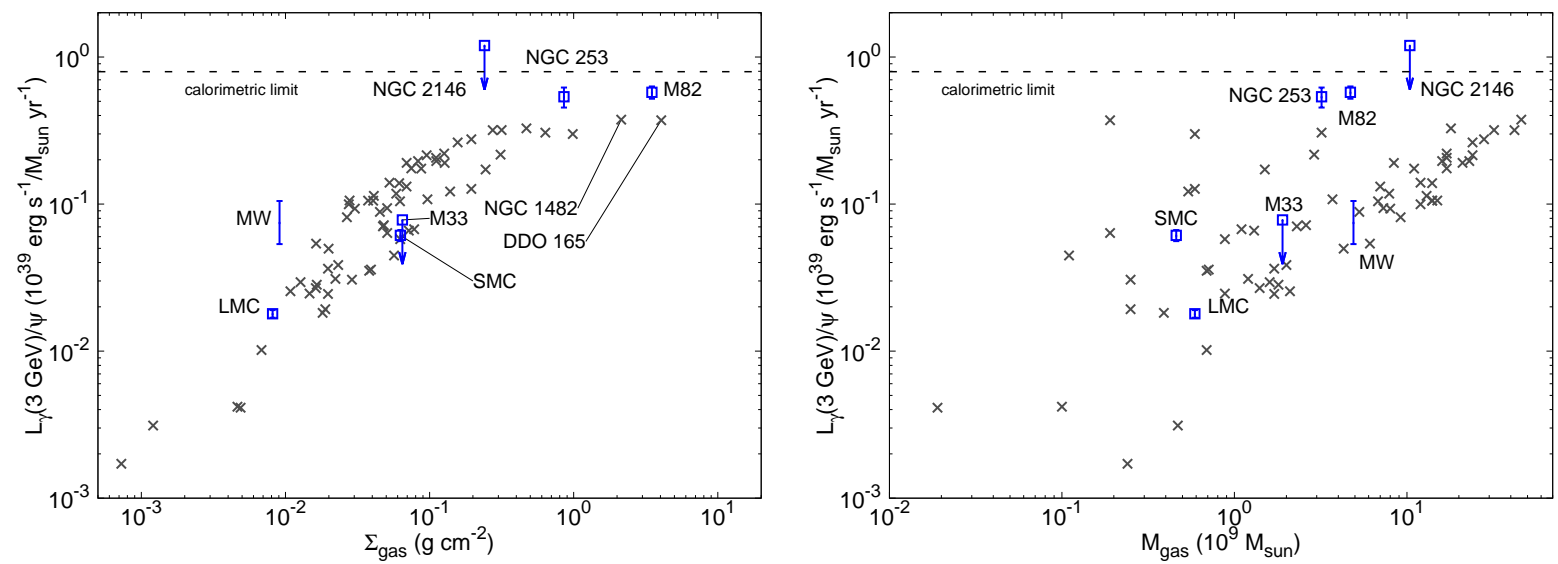

Figure 7. The same as Fig. 6, but for luminosities at $3 \mathrm{GeV}$. The dashed line is the calorimetric limit, $L_{\gamma}(3 \mathrm{GeV}) / \mathrm{SFR} \sim 7.9 \times 10^{38} \mathrm{erg} \mathrm{s}^{-1} / \mathrm{M}_{\odot} \mathrm{yr}^{-1}$ (the same assumption as the TeV calorimetric limit; see Section 3). Blue points are galaxies detected by Fermi-LAT based on the observations by Ajello et al. (2020). The data point for MW is based on Strong et al. (2010), showing the range between the highest and lowest luminosities of their models.

Cherenkov Telescope Array Consortium et al., 2019, Science with the Cherenkov Telescope Array, doi:10.1142/10986.

Chynoweth K. M., Langston G. I., Yun M. S., Lockman F. J., Rubin K. H. R., Scoles S. A., 2008, AJ, 135, 1983

Dale D. A., Helou G., 2002, ApJ, 576, 159

Dale D. A., et al., 2009, ApJ, 703, 517

Diehl R., et al., 2006, Nature, 439, 45

Domingo-Santamaría E., Torres D. F., 2005, A\&A, 444, 403

Eichmann B., Becker Tjus J., 2016, ApJ, 821, 87

Eskew M., Zaritsky D., Meidt S., 2012, AJ, 143, 139

Foley R. J., et al., 2014, MNRAS, 443, 2887

Galametz M., Madden S. C., Galliano F., Hony S., Bendo G. J., Sauvage M., 2011, A\&A, 532, A56

Gonidakis I., Livanou E., Kontizas E., Klein U., Kontizas M., Belcheva M., Tsalmantza P., Karampelas A., 2009, A\&A, 496, 375

Gratier P., et al., 2010, A\&A, 522, A3

H. E. S. S. Collaboration et al., 2018, A\&A, 617, A73

Haynes M. P., et al., 2018, ApJ, 861, 49

Heyer M. H., Corbelli E., Schneider S. E., Young J. S., 2004, ApJ, 602, 723

Holler M., et al., 2015, arXiv e-prints, p. arXiv:1509.02902

Howell J. H., et al., 2007, AJ, 134, 2086

Inoue Y., 2011, ApJ, 728, 11
Inoue Y., Inoue S., Kobayashi M. A. R., Makiya R., Niino Y., Totani T., 2013, ApJ, 768, 197

Israel F. P., 1997, A\&A, 328, 471

Jarrett T. H., Chester T., Cutri R., Schneider S. E., Huchra J. P., 2003, AJ, 125,525

Kelner S. R., Aharonian F. A., Bugayov V. V., 2006, Phys. Rev. D, 74, 034018

Kennicutt Robert C. J., Lee J. C., Funes J. G., J. S., Sakai S., Akiyama S., 2008, ApJS, 178, 247

Kennicutt Robert C. J., et al., 2009, ApJ, 703, 1672

Kennicutt R. C., et al., 2011, PASP, 123, 1347

Knudsen K. K., Walter F., Weiss A., Bolatto A., Riechers D. A., Menten K., 2007, ApJ, 666, 156

Krumholz M. R., Crocker R. M., Xu S., Lazarian A., Rosevear M. T., Bedwell-Wilson J., 2020, MNRAS, 493, 2817

Lacki B. C., Thompson T. A., 2013, ApJ, 762, 29

Lacki B. C., Thompson T. A., Quataert E., 2010, ApJ, 717, 1

Lacki B. C., Thompson T. A., Quataert E., Loeb A., Waxman E., 2011, ApJ, 734,107

Leroy A. K., Walter F., Brinks E., Bigiel F., de Blok W. J. G., Madore B., Thornley M. D., 2008, AJ, 136, 2782

Linden T., Buckman B. J., 2018, Phys. Rev. Lett., 120, 121101 
Lopez L. A., Auchettl K., Linden T., Bolatto A. D., Thompson T. A., Ramirez-Ruiz E., 2018, ApJ, 867, 44

Madden S. C., et al., 2013, PASP, 125, 600

Makiya R., Totani T., Kobayashi M. A. R., 2011, ApJ, 728, 158

Marble A. R., et al., 2010, ApJ, 715, 506

Martin P., 2014, A\&A, 564, A61

Mo H., van den Bosch F. C., White S., 2010, Galaxy Formation and Evolution Montalto M., Seitz S., Riffeser A., Hopp U., Lee C. H., Schönrich R., 2009, A\&A, 507, 283

Moustakas J., Kennicutt Robert C. J., Tremonti C. A., Dale D. A., Smith J.-D. T., Calzetti D., 2010, ApJS, 190, 233

Nieten C., Neininger N., Guélin M., Ungerechts H., Lucas R., Berkhuijsen E. M., Beck R., Wielebinski R., 2006, A\&A, 453, 459

Obreschkow D., Rawlings S., 2009, MNRAS, 394, 1857

Oh S.-H., Brook C., Governato F., Brinks E., Mayer L., de Blok W. J. G., Brooks A., Walter F., 2011, AJ, 142, 24

Paladini R., Montier L., Giard M., Bernard J. P., Dame T. M., Ito S., MaciasPerez J. F., 2007, A\&A, 465, 839

Paturel G., Theureau G., Bottinelli L., Gouguenheim L., Coudreau-Durand N., Hallet N., Petit C., 2003, A\&A, 412, 57

Peng F.-K., Wang X.-Y., Liu R.-Y., Tang Q.-W., Wang J.-F., 2016, ApJ, 821, L20

Peretti E., Blasi P., Aharonian F., Morlino G., 2019, MNRAS, 487, 168

Persic M., Rephaeli Y., Arieli Y., 2008, A\&A, 486, 143

Pfrommer C., Pakmor R., Simpson C. M., Springel V., 2017, ApJ, 847, L13

Pilyugin L. S., Grebel E. K., Kniazev A. Y., 2014, AJ, 147, 131

Rekola R., Richer M. G., McCall M. L., Valtonen M. J., Kotilainen J. K., Flynn C., 2005, MNRAS, 361, 330

Rémy-Ruyer A., et al., 2014, A\&A, 563, A31

Rémy-Ruyer A., et al., 2015, A\&A, 582, A121

Sánchez S. F., et al., 2017, MNRAS, 469, 2121

Sanders D. B., Mazzarella J. M., Kim D. C., Surace J. A., Soifer B. T., 2003, AJ, 126, 1607

Sofue Y., Honma M., Omodaka T., 2009, PASJ, 61, 227

Springob C. M., Haynes M. P., Giovanelli R., Kent B. R., 2005, ApJS, 160, 149

Stanimirovic S., Staveley-Smith L., Dickey J. M., Sault R. J., Snowden S. L., 1999, MNRAS, 302, 417

Staveley-Smith L., Kim S., Calabretta M. R., Haynes R. F., Kesteven M. J., 2003, MNRAS, 339, 87

Strickland D. K., Heckman T. M., Colbert E. J. M., Hoopes C. G., Weaver K. A., 2004, ApJ, 606, 829

Strong A. W., Porter T. A., Digel S. W., Jóhannesson G., Martin P., Moskalenko I. V., Murphy E. J., Orlando E., 2010, ApJ, 722, L58

Sudoh T., Totani T., Kawanaka N., 2018, PASJ, 70, 49

Sudoh T., Linden T., Beacom J. F., 2019, Phys. Rev. D, 100, 043016

Tang Q.-W., Wang X.-Y., Tam P.-H. T., 2014, ApJ, 794, 26

Torres D. F., 2004, ApJ, 617, 966

VERITAS Collaboration et al., 2009, Nature, 462, 770

Wang X., Fields B. D., 2018, MNRAS, 474, 4073

Weiß A., Walter F., Scoville N. Z., 2005, A\&A, 438, 533

Xi S.-Q., Liu R.-Y., Wang X.-Y., Yang R.-Z., Yuan Q., Zhang B., 2020a, ApJ, 896, L33

Xi S.-Q., Zhang H.-M., Liu R.-Y., Wang X.-Y., 2020b, ApJ, 901, 158

Yoast-Hull T. M., Everett J. E., Gallagher J. S. I., Zweibel E. G., 2013, ApJ, 768,53

Yoast-Hull T. M., Gallagher J. S. I., Zweibel E. G., Everett J. E., 2014, ApJ, 780,137

Yoast-Hull T. M., Gallagher John S. I., Aalto S., Varenius E., 2017, MNRAS, 469, L89

Young J. S., Claussen M. J., Kleinmann S. G., Rubin V. C., Scoville N., 1988, ApJ, 331, L81

Young J. S., Xie S., Kenney J. D. P., Rice W. L., 1989, ApJS, 70, 699

Young J. S., et al., 1995, ApJS, 98, 219

van der Marel R. P., 2006, in Livio M., Brown T. M., eds, The Local Group as an Astrophysical Laboratory. pp 47-71 (arXiv : astro-ph/0404192)

\section{APPENDIX A: MODEL PREDICTIONS FOR GALAXIES REMOVED FROM OUR SAMPLE}

In this section, we show the predicted spectra of galaxies removed from our original sample for some reasons (Section 3). Because of the lack of information necessary to make our model predictions, not all of the removed galaxies are considered here.

M31 was removed from our sample due to the discrepancy between the regions of gas and gamma-ray emission. However, the predicted flux and spectrum are in good agreement with observations. This may indicate that the ring-like gas distribution (Ackermann et al. 2017), which is not taken into account in our model, does not seriously affect gamma-ray emission properties.

NGC 1068 and Arp 220, were removed from our sample due to the possibilities of AGN activities. Predicted spectra by our model are lower than observed fluxes, which is consistent if the AGN activities are dominant in gamma-ray emission in these galaxies. Note that the predicted flux would become even smaller for Arp 220 if we consider gamma-ray absorption by pair-production in the galaxy.

This paper has been typeset from a $\mathrm{T}_{\mathrm{E}} \mathrm{X} / \mathrm{L} \mathrm{T}_{\mathrm{E}} \mathrm{X}$ file prepared by the author. 

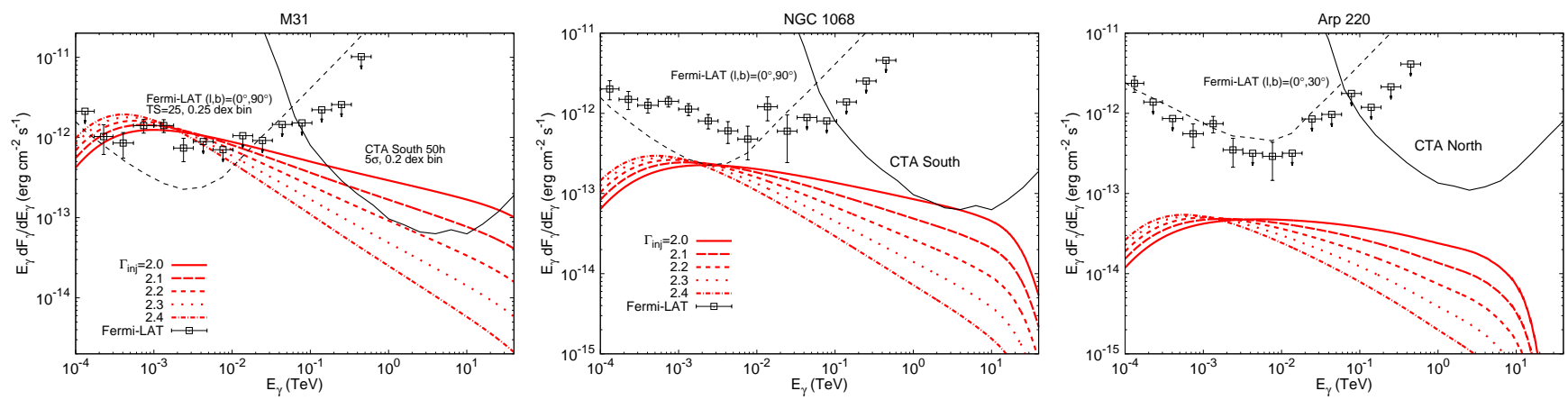

Figure A1. Predicted gamma-ray spectra of M31, NGC 1068, Arp 220 with the observed spectra (Ajello et al. 2020).

Table A1. Physical parameters of M31, NGC 1068, and Arp 220.

\begin{tabular}{ccccccccccc}
\hline name & $D(\mathrm{Mpc})$ & ref & $\psi\left(\mathrm{M}_{\odot} \mathrm{yr}^{-1}\right)$ & ref & $M_{\text {gas }}\left(10^{9} \mathrm{M}_{\odot}\right)$ & ref & $M_{\text {star }}\left(10^{9} \mathrm{M}_{\odot}\right)$ & ref & $R_{\text {eff }}(\mathrm{kpc})$ & ref \\
\hline \hline M31 & 0.79 & $(1)$ & 0.53 & $(1),(2)$ & 7.6 & $(3),(4),(5)$ & 86 & $(6)$ & 1.8 & $(7)$ \\
NGC 1068 & 14 & $(2)$ & 17 & $(2),(8)$ & 11 & $(5),(9),(10)$ & 74 & $(11)$ & 1.5 & $(7)$ \\
Arp 220 & 80 & $(3)$ & 98 & $(2),(12)$ & 51 & $(10),(13),(14)$ & 61 & $(15)$ & 3.5 & $(7)$ \\
\hline
\end{tabular}

References: (1) Kennicutt et al. (2008), (2) Sanders et al. (2003), (3) Braun et al. (2009), (4) Nieten et al. (2006), (5) Pilyugin et al. (2014), (6) Montalto et al. (2009), (7) Jarrett et al. (2003), (8) Adams \& Weedman (1975), (9) Haynes et al. (2018), (10) Young et al. (1995), (11) Howell et al. (2007), (12) Armus et al. (1990), (13) Paturel et al. (2003), (14) Sánchez et al. (2017), (15) Brown et al. (2014),

Table A2. List of gamma-ray fluxes $\left(E_{\gamma} d F_{\gamma} / d E_{\gamma}\right.$ at $1 \mathrm{TeV}$ ) predicted by our model, in units of $\mathrm{erg} \mathrm{s}^{-1} \mathrm{~cm}^{-2}$ assuming $\Gamma_{\text {inj }}=2.2$.

\begin{tabular}{lccccc}
\hline name & $\Gamma_{\text {inj }}=2.0$ & $\Gamma_{\text {inj }}=2.1$ & $\Gamma_{\text {inj }}=2.2$ & $\Gamma_{\text {inj }}=2.3$ & $\Gamma_{\text {inj }}=2.4$ \\
\hline \hline M31 & $2.9 \times 10^{-13}$ & $1.7 \times 10^{-13}$ & $9.2 \times 10^{-14}$ & $4.9 \times 10^{-14}$ & $2.5 \times 10^{-14}$ \\
NGC 1068 & $8.5 \times 10^{-14}$ & $4.9 \times 10^{-14}$ & $2.7 \times 10^{-14}$ & $1.4 \times 10^{-14}$ & $7.2 \times 10^{-15}$ \\
Arp 220 & $2.4 \times 10^{-14}$ & $1.4 \times 10^{-14}$ & $7.4 \times 10^{-15}$ & $3.9 \times 10^{-15}$ & $2.0 \times 10^{-15}$ \\
\hline
\end{tabular}

